\title{
Clinicopathological Analysis of 90 Cases of Polyacrylamide Hydrogel Injection for Breast Augmentation Including 2 Cases Followed by Breast Cancer
}

\author{
Ying Yang $^{\mathrm{a}}$ Shumo Li ${ }^{\mathrm{b}} \quad \mathrm{Jia} \mathrm{He}^{\mathrm{c}}$ Xian Zhao ${ }^{c}$ Wei Chen ${ }^{d} \quad$ Xiaoming Dai $^{\mathrm{c}}$ \\ Liu Liuc \\ ${ }^{a}$ Department of Pathology, The First Affiliated Hospital of Kunming Medical University, Kunming, China; \\ ${ }^{b}$ Department of Breast Surgery, The First Affiliated Hospital of Kunming Medical University, Kunming, China; \\ 'Department of Plastic Surgery, The First Affiliated Hospital of Kunming Medical University, Kunming, China; \\ ${ }^{\mathrm{d}}$ Department of Radiology, The First Affiliated Hospital of Kunming Medical University, Kunming, China
}

\author{
Keywords \\ Polyacrylamide hydrogel injection · Complications · Breast \\ cancer · Breast augmentation
}

\begin{abstract}
Background: Although polyacrylamide hydrogel (PAAG) injection for breast augmentation has been prohibited for many years, the long-term complications will be significant for a long period. Few research articles have focused on the clinicopathological analysis. Materials and Methods: We summarized clinical and pathological features of 90 cases after PAAG-injected breast augmentation, including 2 cases of breast cancer developed following PAAG injection. Results: All patients were females between the ages of 30 and 64 years (mean, 44 years). The complications included masses (75.58\%), pain $(45.35 \%)$, migration $(22.09 \%)$, deformation (18.60\%), infection (16.28\%), induration (4.65\%), and psychological fear (2.33\%). Microscopically, the PAAG presented as purple gel pools, and the foreign body reaction was noted in all 90 patients. The proportion of fibrous component exceeded $90 \%$ in 26 cases $(28.89 \%)$. Chronic and acute inflammation was noted in $70(77.78 \%)$ and $9(10 \%)$ patients, respectively. The mammary gland around the gel displayed atrophy in 18 cases (20.00\%), adenosis in 33 cases (36.67\%), ductal carcinoma in situ in 1 case $(1.11 \%)$, and invasive car-
\end{abstract}

cinoma in 1 case (1.11\%). Conclusion: The long-term complications of PAAG-injected breast augmentation are various and complex. Pathologically, these complications are associated with foreign body reaction, fibrosis, and inflammation.

(c) 2019 S. Karger AG, Basel

\section{Introduction}

Polyacrylamide hydrogel (PAAG) is a combination of a minor backbone of 2.5-5\% cross-linked polyacrylamide and 95-97.5\% apyrogenous water. It has been available for clinical use for more than 40 years for its so-called high biocompatibility, nontoxicity, nonabsorption, and hardness resembling the body's own tissue after injection, and was traditionally used to improve skin contour $[1,2]$. Since 1997, PAAG has been imported from the Ukraine and widely used for breast augmentation in China. In the period from then to 2006 when the State Food and Drug Administration of China administered a full cessation of the clinical injection of PAAG [3], millions of women have received breast augmentation using this material [4]. Long-term complications have been reported including lumps, infection, distant migration, deformation, pain, gel leakage, and difficulty in lactation $[5,6]$. Although PAAG injection has been prohibited in most 
Table 1. Complications after breast augmentation with PAAG

\begin{tabular}{lrr}
\hline Complications & $n$ & $\%$ \\
\hline Mass & 65 & 75.58 \\
$\quad$ Multiple & 58 & 67.44 \\
$\quad$ Single & 7 & 8.14 \\
Detectable by patients & 22 & 25.58 \\
$\quad$ & \\
$\quad$ Detectable by physical or radiological & 43 & 50.00 \\
$\quad$ examination & 39 & 45.35 \\
Pain or feeling uncomfortable & 19 & 22.09 \\
Migration & 16 & 18.60 \\
Deformation & 16 & 18.60 \\
$\quad$ Asymmetry & 1 & 1.16 \\
$\quad 3$ & 3.49 \\
$\quad$ Nipple retraction & 3 & 16.28 \\
$\quad$ Surface depression & 3 & 3.49 \\
Infection & 6 & 6.98 \\
$\quad$ With sinus & 4 & 4.65 \\
$\quad$ Happened in lactation & 2 & 2.33 \\
Induration & 2 & 2.33 \\
Psychological fear & & \\
Malignant tumor & &
\end{tabular}

PAAG, polyacrylamide hydrogel.

countries nowadays, the consequences and long-term complications will be significant for a long period. Many women had to remove the gel by surgery at the cost of losing their breast tissue, and some women had to receive removal surgery more than once. The migration of the gel causes a mass in the abdominal wall or axilla which might mimic malignancy clinically and radiographically, and concealment of the history of breast augmentation because of psychological problems makes the situation more confusing. However, articles and reports on this topic have been published mostly in Russian and Chinese, and most of them focused on the management of patients after PAAG injection. Few pathological analyses have been published. In order to promote the awareness and understanding of surgeons, radiologists, and pathologists, we summarize the clinical and pathological features of 90 cases with complications after PAAG-injected breast augmentation, including 2 cases accompanied by breast carcinoma.

\section{Materials and Methods}

Breast specimens from 90 cases that underwent injected PAAG removal surgery between 2009 and 2017 were collected. The formalin-fixed, paraffin-embedded, hematoxylin and eosin (HE)stained sections were examined under a microscope. The features evaluated under the microscope included the thickness of the capsules, the proportion of the fibrous and cellular components, the density of the fibroblasts, the kind and degree of inflammation, the orientation of the collagen fibrils, and the morphological character of the breast glands involved in the specimens. The clinical and radiological data of these cases were also summarized.

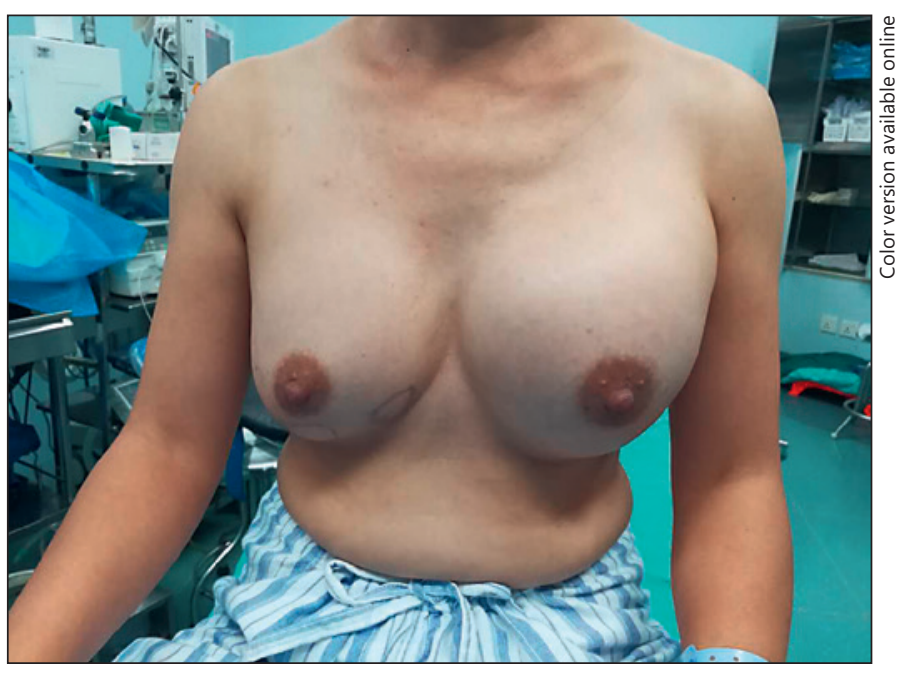

Fig. 1. A 52-year-old woman complained of distending pain in her left breast 12 years after PAAG-injected breast augmentation. Physical examination showed asymmetry in the size and position of the breasts, and the left breast was clearly swollen.

\section{Results}

\section{Clinical Data}

Clinical data were available for 86 patients. All patients were females between the ages of 30 and 64 years (mean, 43.54 years). The age when they received breast augmentation ranged from 18 to 49 years (mean, 32.30 years) with the duration of the implantation from less than 1 year to 20 years (mean, 11.51 years). Seventeen women (19.77\%) had received removal surgery before. The complications are summarized in Table 1. The details are as follows: (1) the most common complication after breast augmentation with PAAG was mass ( $75.58 \%$ of patients). The masses were of medial hardness in texture, irregular in shape, clear in boundary, and had no adhesive to the surrounding tissue under physical examination. (2) The second most common complication was pain or feeling uncomfortable ( $45.35 \%$ of patients). The pain may be distending pain or stabbing pain, intermittent or persistent. Menstruation, fatigue and alcohol consumption may aggravate the pain. The feeling of being uncomfortable included foreign body sensation, feeling of being swollen, and difficulty in breathing. (3) The migration of the gel may present in the infraclavicula, hypochondria, abdominal wall, anterior sternum, axilla, and even the posterior chest wall. (4) Breast deformation occurred in $18.60 \%$ of patients, presenting as asymmetry in the size or position of the breasts, with or without nipple retraction and surface depression (Fig. 1). (5) Infection occurred in 16.28\% of patients, nearly half $(6 / 14)$ of which occurred in the period of lactation. (6) Induration happened in $4.65 \%$ of patients, which means parts of or the whole breast turned 

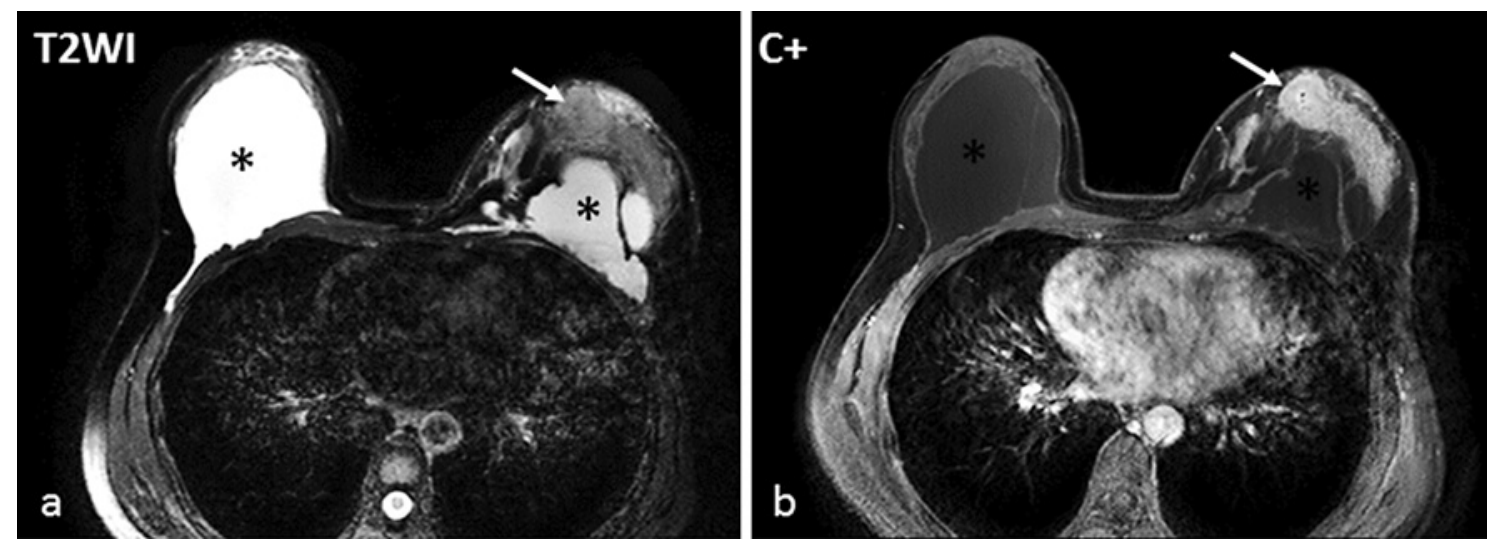

Fig. 2. A 49-year-old female with 12 years of PAAG-injected breast augmentation. a An MRI T2-weighted sequence showed the bilateral breast implants with hyperintensity $\left(^{*}\right)$ and an isointense mass in the left breast (white arrow). b The mass was heterogeneously enhanced with contrast and was histologically validated as a ductal carcinoma in situ (Fig. 3f).

hard or rubbery. (7) Two women (2.33\%) asked for the removal surgery because of psychological fear of potential harm to the body or possible difficulty in lactation without any symptom or sign. (8) Two women (2.33\%) had malignant tumors. One was a 49 -year-old woman complaining of a mass in her left breast for 12 years and increasing in size for 7 months; the other was a 47 -year-old woman complaining of pain in her left breast for half a month. The duration of the implantation was 12 years in both cases. Fifty-two patients $(60.47 \%)$ had two or more complications.

Ultrasonography was available in 73 (84.88\%) women. The injected PAAG appeared as an anechoic or hypoechoic collection with internal foci of varying echogenicity, accompanied with hypoechoic or heteroechoic nodules, one of which was suspected as malignant (confirmed as a gel nodule by pathology). Magnetic resonance imaging (MRI) was performed in 30 patients (34.88\%) and depicted the location of the injected gel accurately. PAAG showed hypointense on T1-weighted and hyperintense on T2-weighted sequences. Mammograms were available in 11 patients (12.79\%). The polyacrylamide gel showed an amorphous mass with water density in mammography, and oval opacity could be seen in 6 patients. One of opaque nodules was suspected as malignant which turned out to be a gel nodule. For the 2 cases accompanied by malignant lesions, MRI revealed a mass with BI-RADS $4 \mathrm{C}$ in one case (Fig. 2), and radiological examination failed to identify the 2-mm malignant lesion in the background of PAAG and infection in the other case.

During the removal operation, the majority of PAAG was present in the retromammary space in 82 patients $(95.35 \%)$ while in 4 patients (4.65\%) it was in the subpectoral space. The gel diffused extensively into the mammary gland, fatty tissue, and intramuscularly into the pec- toralis major and minor muscles in 61 patients (70.93\%). In 2 cases $(2.33 \%)$ the gel diffused into the intercostal muscles. The PAAG was confined to the retromammary space in only $10(11.63 \%)$ cases. The volume of the gel removed unilaterally ranged from 30 to $700 \mathrm{~mL}$.

\section{Pathological Findings}

Grossly, the gel was sticky or grainy, mixed with some firm or fragile nodules. The cut surfaces of the nodules showed a translucent yellow or milky-white gelatinous appearance with varying amounts of gray area. Microscopically, the PAAG presented in HE sections as pale-todark purple homogenous gel pools of various sizes and shapes. Most of the gel pools were shaped round to oval, but some appeared ragged. Most gel pools were located in the stroma, while others were closely adjacent to breast glands, muscles, and fat tissues (Fig. 3a). The foreign body reaction was noted in all 90 patients. There were two relationship patterns between macrophages and gel pools: (1) some macrophages and multinucleated foreign bodytype giant cells closely surrounded the gel pool (Fig. 3b), and (2) Some macrophages entered and traversed the gel pools, engulfed the gel, and formed purple inclusions within the cytoplasm. In some cases, the number of macrophages that entered the gel was so huge that the gel pool presented as an extraordinary cellular area, in which the remnant gel simulated the extracellular matrix of macrophages and multinucleated giant cells (Fig. $3 \mathrm{c}$ ). The two patterns mixed together with various proportions in different cases, and in 13 cases (14.44\%) the cellular pattern covered over $50 \%$ of the tissues. The foreign body reaction was subtle in 14 cases $(15.56 \%)$ where the almost completely unreactive connective tissue with interdigitating strands of gel was seen (Fig. 3d). 

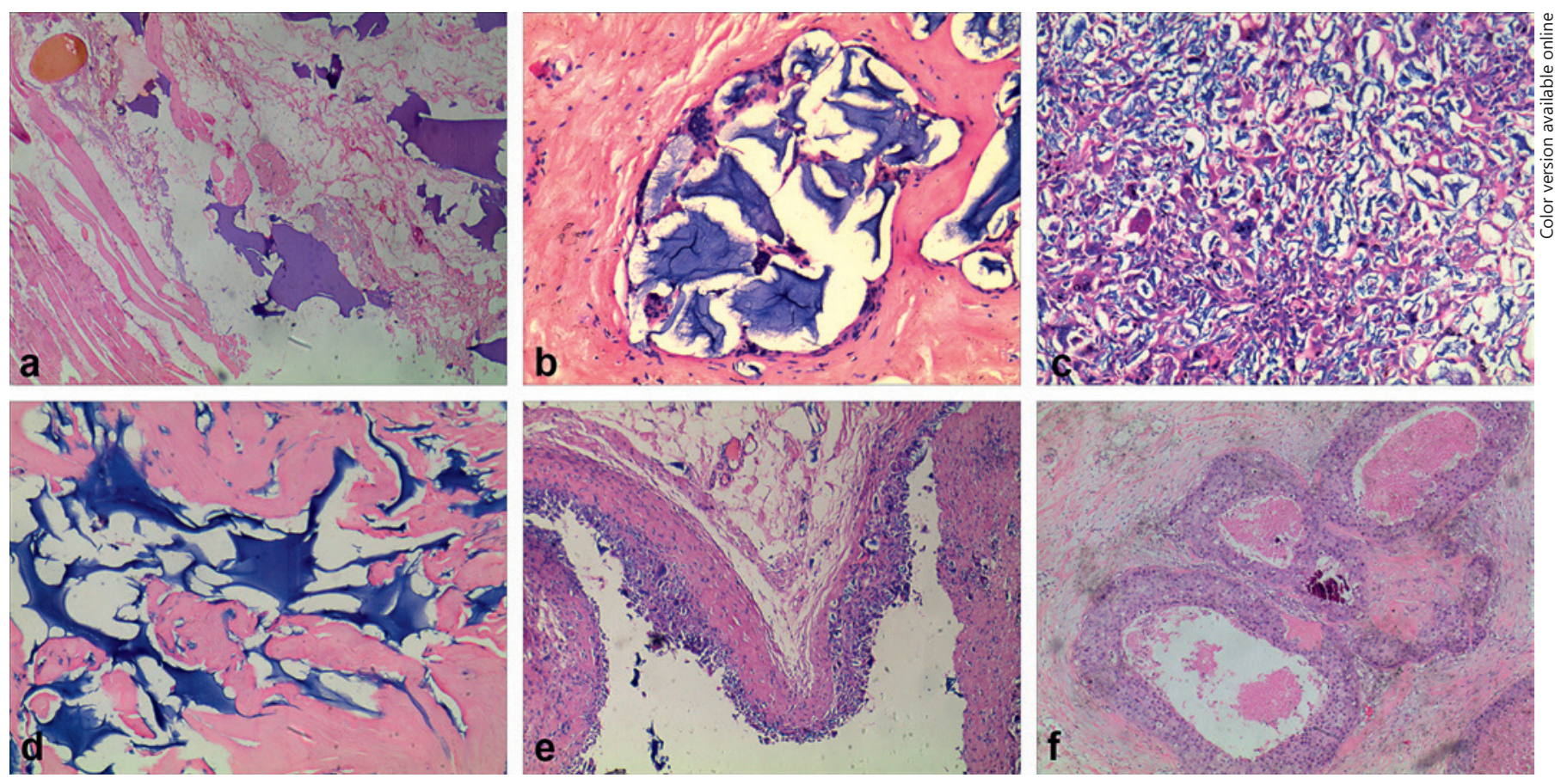

Fig. 3. Histological features of PAAG injection for breast augmentation. a Some PAAG gel pools were closely adjacent to muscles and fat. HE. $\times 40$. $\mathbf{b}$ Macrophages and multinucleated foreign body-type giant cells closely surrounded the gel pool. HE. $\times 40$. c Macrophages entered the gel pools and presented as an extraordinary cellular area. HE. $\times 100$. $\mathbf{d}$ The foreign body reaction was subtle in some areas. HE. $\times 100$. e The typical pseudocapsule was composed of an inner cellular layer and an outer fibrous layer. HE. $\times 40 . \mathbf{f}$ DCIS arose from a PAAG-injected breast. HE. $\times 40$.

The extent of fibrosis varied in different cases. The proportion of fibrous component exceeded $90 \%$ in 26 cases $(28.89 \%)$. The pseudocapsule around the gel fillers was noted in 56 cases (62.22\% of patients). The capsule was either incomplete or inconspicuous with a thickness ranging from $10 \mu \mathrm{m}$ to $2 \mathrm{~mm}$. The typical pseudocapsule was composed of an inner cellular layer and an outer fibrous layer. The inner layer at the capsule-implant interface presented as a loose mixture of macrophages, multinucleated giant cells, and gel, while the outer layer was composed of layers of fibers of differing fiber density, thickness, and alignment (Fig. 3e). The collagenous fibers were oriented parallel to the long axis of the capsule in 55 cases (98.21\% of the patients with pseudocapsule). The density of the fibroblast in the capsule ranged from low to medium. Calcification of the pseudocapsule was found in 8 cases $(8.89 \%)$.

Chronic inflammation was noted in 70 patients (77.78\%), characterized by infiltration of lymphocytes and plasma cells; 55 of them $(78.57 \%)$ were mild. Acute inflammation with neutrophilic infiltration was seen in 9 patients $(10.00 \%), 2$ of which were accompanied by abscesses.

The mammary gland around the gel displayed atrophy in 18 cases $(20.00 \%)$, adenosis in 33 cases $(36.67 \%)$, fibro- adenoma in 1 case (1.11\%), ductal carcinoma in situ (DCIS) with microinfiltration in 1 case $(1.11 \%)$, and invasive carcinoma in 1 case $(1.11 \%)$. The DCIS displayed a solid growth pattern and high-grade nucleus (Fig. 3f). The invasive carcinoma was well differentiated (grade 1) with a maximum diameter of $2 \mathrm{~mm}$, accompanied by micropapillary DCIS.

\section{Relationship between Complications and Pathological Findings}

Patients who experienced masses seemed more likely to present obvious fibrosis microscopically (defined as the proportion of fibrous component exceeding 90\%) compared with patients without mass (30.77 vs. $24.00 \%$ ). Similarly, the patients with pain were more likely to present obvious fibrosis ( 38.46 vs. $21.57 \%$ ), while the patients with gel migration were less likely to present obvious fibrosis ( 10.53 vs. $33.8 \%$ ). However, the differences did not achieve statistical significance. A significant difference was observed in acute inflammation microscopically between patients with and without infection complication (64.29 vs. $1.32 \%, p<0.05)$. 


\section{Discussion}

Although PAAG injection has been prohibited for about 10 years, patients who have received PAAG-injected breast augmentation come to seek medical advice ceaselessly because of complications or psychological fear. Since the material is injected blindly, the outcome is unpredictable. The diversity of the injection layer, the uncertainty of the gel migration, and the variety of the patients' reactions determined the complexity of the complications, pathological characters, and surgery methods [7].

It is difficult to know the true rate of PAAG injectionrelated complications since no prospective study examined the complications associated with PAAG injection, and the data from a few retrospective studies varied considerably. The manufacturer of one brand of PAAG claimed that one in every 1,500 patients experiences transient swelling and tenderness [5], while two Chinese articles reported the rates as $1.44 \%(12 / 833)$ and $18.30 \%(262 / 1,432)$ [8]. Christensen et al. [1] reported that of the approximately 300 women who underwent PAAG-injected breast augmentation, 27 (9.0\%) cases returned for reoperation, omitting the cases with minor complications that needed no surgical treatment. Short-term reactions included lumps, hematomas, and infections [5]. The most common long-term complication was found to be mass as our series shows. The rates reported ranged from 22 to $79 \%[1,9]$. Other long-term complications include pain, induration, infection, injection migration, breast deformity, galactorrhea, late hematoma, seroma, and galactocele, among others [4, 10].

Radiological examination has an important role in the evaluation of women with PAAG mammoplasty. The radiological appearance is nonspecific but becomes easily recognizable when proper history is obtained and when the radiologist is aware of this entity. Ultrasound is a useful tool for providing guidance for the location of the gel nodules and for aspiration of the inflamed collection, while MRI depicts with greater ease and accuracy the exact location of the injected gel and is thought to be the most sensitive technique for assessing the volume and distribution of PAAG [11]. The gel nodules may mimic malignancy clinically and radiographically, and the hyperdense gel and lumps may interfere with the detection of malignant lesions. Moreover, the dystrophic calcification of the pseudocapsule in rare cases may further complicate the identification of malignancy. The American College of Radiology recommends breast MRI for cancer screening in patients with a history of breast augmentation, including silicone and PAAG [12].

The foreign body reaction, fibrosis, and inflammation are the basic pathological elements of the breast after PAAG-injected augmentation. They are associated with various complications. As a polymer of firmly and irreversible bound monomers, PAAG is widely resistant to degra- dation and phagocytosis by circulating macrophages. Instead, the macrophages enter and traverse the gel and are replaced by a scaffold of thin connective tissue fibers [13]. These theories are consistent with the microscopic features in our series. As demonstrated by other studies, no thick capsule like that around an implant has been investigated in our series. The real capsule around an implant is composed of multiple layers of fibers, with low cellularity and a layer of synovial-like cells arranged at the capsule-implant interface [14], while the pseudocapsule around PAAG consists of gel and surrounding macrophages in the inner layers and thin fibers in the outer layers. Yu et al. [15] named the pseudocapsule as an infiltrate capsule and strongly recommended its removal during surgery.

Although acrylamide has inherent toxicity including neurotoxicity, genotoxity, and carcinogenicity, and is classified as a group 2A substance by the International Agency for Research on Cancer and a category 2 mutagen by the European Union [16], no toxic reaction was observed when administered orally to rats, fish, and dogs as long as its content was below $0.0064 \mu \mathrm{g} / \mathrm{ml}$, nor were complications encountered when it was inhaled by workers during polyacrylamide production [17]. However, Xi et al. [18] found that PAAG induces the increase of mRNA expression of $c-m y c$, inhibits the growth of fibroblasts, causes the apoptosis of fibroblasts, and alters the physical parameters of these cells. Three cases of breast cancer occurring after PAAG-injected breast augmentation have been reported $[19,20]$ apart from one invasive carcinoma and one DCIS in our series. Although there is no direct evidence that PAAG causes malignant lesions, there is the possibility that the breast induration and mass caused by PAAG could delay the early screening of malignancy, and the confusing radiological features could interfere with the detection of malignancy. Surgeons and pathologists must be more aware of possible breast cancer in patients after PAAG injection for breast augmentation.

\section{Statement of Ethics}

The authors state that the subjects provided their written informed consent. The study protocol was approved by the Medical Ethics Committee of Kunming Medical University.

\section{Disclosure Statement}

The authors have no conflicts of interest to declare.

\section{Funding Sources}

This work was supported by the National Natural Science Foundation of China (grant No. 81460298) and the Scientific Research Foundation of the Education Department of Yunnan Province (grant No. 2017YJS083). 


\section{References}

1 Christensen LH, Breiting VB, Aasted A, Jørgensen A, Kebuladze I. Long-term effects of polyacrylamide hydrogel on human breast tissue. Plast Reconstr Surg. 2003 May;111(6): 1883-90.

2 Wolters M, Lampe H. Prospective multicenter study for evaluation of safety, efficacy, and esthetic results of cross-linked polyacrylamide hydrogel in 81 patients. Dermatol Surg. 2009 Feb;35(Sup 1 Suppl 1):338-43.

3 China Food and Drug Administration [cited 2010 September 03]. Available from: http:// samr.cfda.sda.gov.cn/WS01/CL1994/53328. html.

4 Chen B, Song H. Management of breast deformity after removal of injectable polyacrylamide hydrogel: retrospective study of 200 cases for 7 years. Aesthetic Plast Surg. 2016 Aug;40(4):482-91.

5 Ono S, Ogawa R, Hyakusoku H. Complications after polyacrylamide hydrogel injection for soft-tissue augmentation. Plast Reconstr Surg. 2010 Oct;126(4):1349-57.

6 Unukovych D, Khrapach V, Wickman M, Liljegren A, Mishalov V, Patlazhan G, et al. Polyacrylamide gel injections for breast augmentation: management of complications in 106 patients, a multicenter study. World J Surg. 2012 Apr;36(4):695-701.
7 Patlazhan G, Unukovych D, Pshenisnov K. Breast reconstruction and treatment algorithm for patients with complications after polyacrylamide gel injections: a 10-year experience. Aesthetic Plast Surg. 2013 Apr;37(2): 312-20.

8 Cheng NX, Wang YL, Wang JH, Zhang XM, Zhong H. Complications of breast augmentation with injected hydrophilic polyacrylamide gel. Aesthetic Plast Surg. 2002 Sep-Oct;26(5): 375-82.

9 Luo SK, Chen GP, Sun ZS, Cheng NX. Our strategy in complication management of augmentation mammaplasty with polyacrylamide hydrogel injection in 235 patients. J Plast Reconstr Aesthet Surg. 2011 Jun;64(6): 731-7.

10 Cheng NX, Zhang YL, Luo SK, Zhang XM, Hui L, Chen YL, et al. Late hematoma, seroma, and galactocele in breasts injected with polyacrylamide gel. Aesthetic Plast Surg. 2011 Jun;35(3):365-72.

11 Khedher NB, David J, Trop I, Drouin S, Peloquin L, Lalonde L. Imaging findings of breast augmentation with injected hydrophilic polyacrylamide gel: patient reports and literature review. Eur J Radiol. 2011 Apr;78(1):104-11.

12 Margolis NE, Bassiri-Tehrani B, Chhor C, Singer C, Hernandez O, Moy L. Polyacrylamide gel breast augmentation: report of two cases and review of the literature. Clin Imaging. 2015 May-Jun;39(3):339-43.

13 Christensen L. Normal and pathologic tissue reactions to soft tissue gel fillers. Dermatol Surg. 2007 Dec;33 Suppl 2:S168-75.
14 Bui JM, Perry T, Ren CD, Nofrey B, Teitelbaum S, Van Epps DE. Histological characterization of human breast implant capsules. Aesthetic Plast Surg. 2015 Jun;39(3):306-15.

15 Yu L, Wang J, Zhang B, Zheng DN, Zhu C. Treatment of breast injection with polyacrylamide hydrogel with infiltrated fascia capsule removal: report on 104 cases. Aesthetic Plast Surg. 2012 Oct;36(5):1120-7.

16 Dybing E, Farmer PB, Andersen M, Fennel TR, Lalljie SP, Müller DJ, et al. Human exposure and internal dose assessments of acrylamide in food. Food Chem Toxicol. 2005 Mar;43(3):365-410.

17 Leung KM, Yeoh GP, Chan KW. Breast pathology in complications associated with polyacrylamide hydrogel (PAAG) mammoplasty. Hong Kong Med J. 2007 Apr;13(2): 137-40.

18 Xi TF, Fan CX, Feng XM, Wan ZY, Wang CR, Chou LL. Cytotoxicity and altered c-myc gene expression by medical polyacrylamide hydrogel. J Biomed Mater Res A. 2006 Aug;78(2): 283-90.

19 Chen G, Wang Y, Huang JL. Breast cancer following polyacrylamide hydrogel injection for breast augmentation: A case report. Mol Clin Oncol. 2016 Mar;4(3):433-5.

20 Cheng NX, Liu LG, Hui L, Chen YL, Xu SL. Breast cancer following augmentation mammaplasty with polyacrylamide hydrogel (PAAG) injection. Aesthetic Plast Surg. 2009 Jul;33(4):563-9. 